\title{
SECADO SOLAR TÉCNICO DE LUCUMA (Lúcuma ovobata H.P.K.)
}

\author{
Enrique De Florio Ramirez'; Arturo Figueroa Garcia ${ }^{2}$; Edgard Guido Calderón Copa ${ }^{3}$ Linley Vega Vega
}

\section{RESUME N}

El secado de alimentos es el proceso más utilizado en la conservación de alimentos consistiendo en la remoción de gran parte del agua inicial. El rendimiento de la lúcuma fue: peso total de Materia Prima 581 gr, peso de la cáscara más pepa 213 gr., peso de la Pulpa 368 gr. y el rendimiento de Materia Prima 63.33\%, más bajo que el obtenido para la variedad giraldo, debido al pelado la fruta.

Las caracteristicas organolépticas del producto final fueron que seguia teniendo el color, olor; sabor característico, textura dura, los realizados con un panel semi entrenado, dan que no existen diferencias significativas entre el sabor de las muestras obtenidas por secado solar tradicional y el secado solar técnica.

En operaciones preliminares para el secado, el sulfitado no es necesario, no presentó alteración de color. El porcentaje de humedad final (9.26\%), obtenido para el secado solar técnico fue adecuado. En valores de humedad cercanos a la Humedad de Equilibrio del producto se establece una serie de estados de hidratación (noche) y deshidratación (dia).

\section{A BSTRACT}

The drying of foods is the process more used in the conservation of foods consisting on the removal of great part of the initial water. The yield of the lúcuma was: I weigh total of Matter 581 gr Prevails., I weigh of the shell more pepa $213 \mathrm{gr}$., I weigh of the Pulp $368 \mathrm{gr}$ and the yield of Matter Prevails $63.33 \%$, 10 wer than the one obtained for the variety giraldo, due to the peeled one the fruit.

The characteristic organolepticas of the final product was that it continued having the color, scent, characteristic flavor, hard texture, those carried out with a panel trained semi, give that significant differences don t exist among the flavor of the samples obtained by drying traditional lot and the technical solar drying.

In preliminary operations for the drying the sulfitado is not necessary, it didn't present color alteration. The percentage of final humidity (9.26\%), obtained for the technical solar drying it was adapted. In near values of humidity to the Humidity of Balance of the product a series of hydrate states settles down (night) and dehydration (day).

\section{INTRODUCCIÓN}

El Perú es un pais con pocas tierras aptas para la agricultura y de baja productividad, principalmente en la zona andina, originándose la carencia del recurso alimentario y el empobrecimiento del poblador rural.

La infraestructura vial entre la sierra, la selva y la costa se encuentra en pésimo estado u otras veces es inexistente.

En muchas zonas rurales se carece de energía eléctrica y el acceso es difícil y costoso el suministro de combustible. Agregándose a esto la inexistencia de infraestructura de almacenamiento.

Asímismo, en paises en vías de desarrollo se reporta una pérdida en el $50 \%$ de la cosecha en frutas y hortalizas por efecto de la carencia de una infraestructura post-cosecha adecuada que vaya descle la cosecha hasta el consumidor (Salas, 1989).

El desarrollo de técnicas post cosecha, almacenamiento, conservación y la producción y mezclas de harinas y mezclas alimenticias serían un salto importante en el logro de mayor productividad y una política alimentaría adecuada (Carazo, 1986).

\section{PLANTEAMIENTO DE LA INVESTIGACIÓN}

\section{DESCRIPCIÓN DEL PROBLEMA}

Dentro de los productos agrícolas que tienen un potencial de industrialización alto, se encuentra la Lúcuma (Lúcuma obovata H.P.K.), según lo sostiene

(1) (2) Ingeniero en Industrias Alimentarias

(3) Quimico Farmaceutico

(4) Bachiller en Industrias Alimentarias 
Brenner en 1965, de está se elabora cierto tipo de harina que es utilizada como saborizante, principalmente en la industria del helado.

El fruto tiene una alta perecibilidad y tiende a descomponerse rápidamente, aún en las mejores condiciones después de madurarse, además tiene una piel muy deigada lo cuál la hace muy vulnerable a los esfuerzos mecánicos que se producen durante el manipuleo y transporte, desde la cosecha al consumidor.

\section{ANTECEDENTES DEL PROBLEMA}

La consistencia pastosa de la pulpa dificulta su utilización en fresco en industrias como pastelerfa, postres preparados de uso directo, aunque existe la investigación realizada por Aliaga en 1979, sobre Néctar, jalea, trozos de lúcuma en almibar, los cuales reportan con resultados satisfactorios, pero indicando que el néctar tenía una consistencia áspera debido posiblemente a los almidones en suspensión.

Cisneros en 1959, sostiene que uno de los mayores inconvenientes que restringen su consumo es la dificultad de conservación en el mercado.

\section{MATERIALES Y MÉTODOS}

El presente trabajo de investigación se realizó en los siguientes lugares: Laboratorios de FAIA-UNJBGTacna, Laboratorio de Secado Solar de la UNJBG-Tacna y Laboratorio de Cómputo F.A.I.A.

\section{MATERIALES}

\section{MATERIA PRIMA}

Lúcuma (Lúcuma obovata H.P.K.), obtenida en el mercado local de procedencia de la Subregión Moquegua.

\section{MATERIALES}

Envases de polietileno transparente, cuchillos de Acero Inoxidable, espátula, Bandejas, Mesas de trabajo, reloj, Luna de reloj, Balanza analítica.

\section{EQUIPO}

Banco de Secado Solar del Técnico Centro de investigación de Energías no renovables de la UNJBGTacna.

Otros Equipos. Anemómetro digital marca POWER INSTRUMENTAL, Balanza de Pesal AHEWA Cap 5 kg., Termohidrométro de cuerda, marca THIES GOTTING Germany.

\section{METODOLOGÍA}

Los procedimientos a seguir consistió en las siguientes etapas:

\section{EXPERIMENTO EN SECADO}

El flujo de operaciones que se seguirán, se muestra en la fig. $N^{\circ} 5$ y consta de las siguientes operaciones:

Selección: Se separarán los productos que presenten deterioro y el grado de madurez óptimo.

Lavado-desinfectado- - Utilizando una solución con detergente, para remover impurezas y eliminar contaminantes. Esta se realizará en forma manual con agua potable.

Pelado y Corte.- Se realizará en forma manual, se eliminarán cáscaras y pepas. La pulpa se cortará en pequeñas planchas de $5 \mathrm{~mm}$ de espesor.

Sulfitado.-Inmediatamente después de pelado a una de las muestras se le sumergirá en una solución de Bisulfato de sodio por espacio de 5 minutos, y (Saenz $\mathrm{H}$, Carmen, et a1) y a otra no, luego se llevarán por espacio de 24 horas al medio ambiente para determinar la presencia de pardeamiento enzimático, por la conservación visual del cambio de color.

Con la muestra que mejor resultado se obtenga se continuará el proceso.

Secado.- la muestra se lleva al secador solar técnico mixto de cabina

Durante ei secado solar se tomarán los pesos la muestra durat:ce las horas de sol, para asi obtener las curvas de seciado.

Asímismo, se colocará una muestra al medio ambiente para obtener una muestra a utilizarse en el análisis organoléptico.

Envasadc.- Las muestras se colocarán en envases de plástico transparente herméticamente cerrados.

Almacenado.- Se almacenará a temperatura ambiente.

Figura 05: Diagrama de Operaciones del Secado de Lúcuma

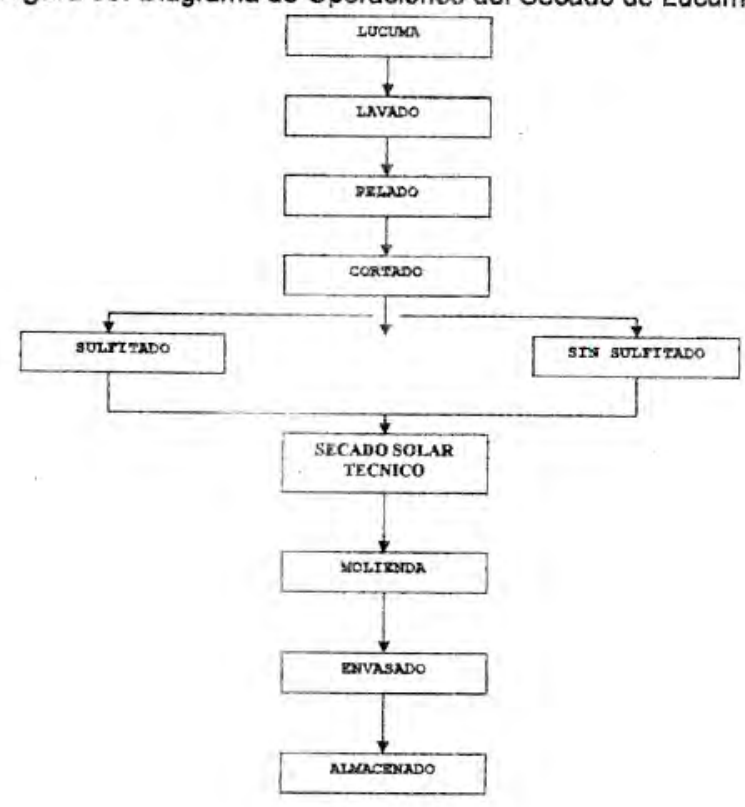




\section{ANÁLISIS EN EL. PRODUCTO}

$\rightarrow$ Humedad del producto: Método de A.D.A.C. (1980).

$\rightarrow$ Color (visual).

\section{MEDICIONES DURANTE ELSECADO}

\section{En el Aire de Secado.-}

Temperatura de Bulbo seco y bulbo húmedo del aire de entrada al secador serán obtenidas en la Estación Agrícola de los "Pichones" de SENAIM. Ubicada dentro del Perimetro de la Ciudad Universitaria de la UNJBB. Las coordenadas de estas son: Lat. $10^{\circ}, \log 70^{\circ} 15^{\circ}$ altitud $425 \mathrm{msnm}$. Los valores promedios estimados de humedad, velocidad, dirección del viento, asi como las horas de sol serán obtenido de promedios de la serie histórica de 10 años para el presente mes, obtenidos de la Estación de Poliometereológia de Calana con las siguientes coordenadas: latitud $10^{\circ} 10^{\prime}$, longitud $70^{\circ} 15^{\circ}$, altitud $525 \mathrm{msnm}$.

La velocidad, temperatura y humedad del aire de salida de cámara de secado, se controlará con el anemómetro y el termohidrómetro respectivamente, colocándolo en la boca interna de la chimenea de la cámara de secado.

\section{En el Producto.-}

Peso de la muestra cada hora para determinar la velocidad del secado y la pérdida de humedad a través del tiempo de duración del secado, el que se considerará cuando el valor del registro de peso se vuelva una cantidad constante. Humedad del producto al inicio y final operación de secado.

\section{EXAMENES ORGANOLÉPTICOS}

Con el producto seco se elaborará una harina la que se adicionará en una cantidad de $2 \mathrm{gr}$. A $100 \mathrm{gr}$. De yogurt natural previamente edulcorado. Se prepararán dos muestras a partir de la adición de las siguientes harinas.

A: Harina de lúcuma de la muestra colocada en el secador solar técnico de cabina.

B : Harina de lúcuma obtenida de la muestra colocada al medio ambiente.

Estas muestras serán presentadas a un panel semientrenado para su evaluación organoléptica del sabor mediante una escala Hedónica de 5 purtos. Esta última y el formato para el registro del análisis de cada panelista se encuentran respectivamente. El diseño estadístico que se utilizará será el de Bloques Completos al azar.

\section{RESULTADO Y DISCUSIÓN}

El rendimiento de la lúcuma fue le siguientes:

Peso total de Materia Prima $581 \mathrm{gr}$.

Peso de la cáscara más pepa $213 \mathrm{gr}$.

Peso de la Pulpa (por diferencia) $368 \mathrm{gr}$.

Rendimiento de la Materia Prima $63.33 \%$.

Este rendimiento es ligeramente más bajo que el obtenido para la variedad giraldo y se debe a que en pelado la fruta se adhería a la cáscara generando un alto porcentaje en pérdidas aproximado $37 \%$ que es más alto que el proporcionado por el INIDA (18\%).

La humedad inicial del producto fue aproximadamente de $66.5 \%$ que es cercana a la que obtuvo Brenner (1965).

Las características externas del fruto son:Cáscara de la pulpa; color amarillo, sabor y olor caracteristico, textura pastosa al tacto.

La temperatura de entrada del aire al secador se muestra en el cuadro $N^{\circ} 04$ y la temperatura de salida del aire de la cámara del secador solar técnico, en el cuadro $N^{\circ} 5$. Al respecto se puede decir que guardan una estrecha alcanzándose a las 13 hrs una temperatura del aire ambiental.

El veior más alto de temperatura dentro de la cámara del secsdo es $47^{\circ} \mathrm{C}$ (máxima alcanzada) y $30^{\circ} \mathrm{C}$ (minima de trabajo), como se puede apreciar en ambos cuadros. En el medio ambiente la temperatura varía entre $25^{\circ} \mathrm{C}$ (max) y $14^{a} \mathrm{C}(\mathrm{min})$. Cuadro $\mathrm{N}^{\circ} 4$

Con respecto a la velocidad del aire en la Cámara de secado, es casi. Nula no registró valores (,movimiento del aire por convección).

\begin{tabular}{|c|c|c|c|c|c|}
\hline Dáa & $\begin{array}{l}\text { Tiempo } \\
\text { (ths) }\end{array}$ & $\begin{array}{l}\text { Temperatura } \\
\text { Bulbo Seco }\end{array}$ & $\begin{array}{l}\text { Temperature } \\
\text { Bulbo Hamedo }\end{array}$ & $\begin{array}{l}\text { Temperatura } \\
\text { Maxima }\left({ }^{\circ} \mathrm{C}\right)\end{array}$ & $\begin{array}{l}\text { Temperatura } \\
\text { Minima }\left({ }^{\circ} \mathrm{C}\right)\end{array}$ \\
\hline \multirow{3}{*}{01} & - & & & 248 & 14.7 \\
\hline & 07 & 15.2 & 13.6 & - & $\rightarrow$ \\
\hline & 13 & 25.2 & 18.6 & - & $\rightarrow$ \\
\hline \multirow{4}{*}{02} & $\frac{18}{-}$ & $16 \mathrm{~s}$ & $\frac{144}{-}$ & $\overline{25}$ & $\overline{14.0}$ \\
\hline & 07 & 16.8 & 13.1 & - & - \\
\hline & 13 & 26.5 & 17.3 & - & - \\
\hline & 10 & 122 & 14.2 & - & - \\
\hline \multirow{4}{*}{03} & $=$ & - & - & 23 & 145 \\
\hline & $\sigma r$ & 15.5 & 13.6 & - & - \\
\hline & 13 & 21.8 & 16.2 & - & - \\
\hline & 18 & 18.2 & 15.0 & - & - \\
\hline
\end{tabular}

En relación a las operaciones preliminares al secado se pueqe Jecir que se presentó cierta dificultad en el pelad a y cortado por la consistencia pastosa del producto, pegándose pulpa a la cáscara, la que también disminuyó el rendimiento de fruta en pulpa.

En cuanto a la operación de sulfitado no se presentó alteración de color en ambas muestras.

Los resultados de la evaluación de la operación de secado se muestran en el cuadro $\mathrm{N}^{\circ} 6$ y gráfico $\mathrm{N}^{\circ} 6$, $6 a, 7,8$. 
En el Cuadro $N^{\circ} 6$ se puede observar que las muestras se encontraron por espacio de 47 horas en el secador, pero realmente fueron 16 horas las que realizó el secado, donde no se consideran las horas de noche, ni los perlodos de eliminación de agua absorvida por efecto de la rehidratación.

Cuadro $N^{\circ}$ 5: Temperatura, humedad y velocidad del aire en la cabina del s ecador solar técnico de cabina mixto

\begin{tabular}{|c|c|c|c|c|}
\hline Dia & $\begin{array}{c}\text { Tiempo } \\
\text { ( } \mathrm{H} \mathrm{rs})\end{array}$ & Temperatura $\left({ }^{\circ} \mathrm{C}\right)$ & $\begin{array}{c}\text { Humedad Relativa } \\
(\%)\end{array}$ & $\begin{array}{l}\text { Velocidad del aire } \\
\text { (Pie/min) }\end{array}$ \\
\hline \multirow{6}{*}{01} & 11.30 & 44 & 34 & S/R \\
\hline & 12.30 & 43 & 35 & Idem \\
\hline & 13.30 & 43 & 35 & Idem \\
\hline & 14.30 & 40 & 38 & Idem \\
\hline & 15.30 & 39 & 40 & Idem \\
\hline & 16.30 & 32 & 45 & Idem \\
\hline \multirow{9}{*}{02} & 09.00 & 43.5 & 80 & Idem \\
\hline & 10.00 & 43.5 & 38 & Idem \\
\hline & 11.00 & 46 & 35 & Idem \\
\hline & 12.00 & 46.5 & 32 & Idem \\
\hline & 13.00 & 60 & 31 & Idem \\
\hline & 14.00 & 46 & 33 & Idem \\
\hline & 15.00 & 45 & 35 & Idem \\
\hline & 16.00 & 32 & 38 & Idem \\
\hline & 17.00 & 30 & 44 & Idem \\
\hline \multirow{4}{*}{03} & 08.30 & 20 & 80 & Idem \\
\hline & 09.30 & 43 & 38 & Idem \\
\hline & 10.30 & 45 & 35 & Idem \\
\hline & 11.30 & 46 & 35 & Idem \\
\hline
\end{tabular}

En los gráficos 6 y $6 \mathrm{~A}$ se observa que muestra una tendencia típica. En el cuadro 6, indica una disminución gradual de la humedad. Si se observa el eje de las " $X$ " se notará que está seccionado a horas de los casos es una recta en partes iniciales de equilibrio se rehidrata, por acción de diferencias de presiones de vapor del producto y el medio circundante y se reinician a las 8 hrs.

Una vez liegado a la humedad de equilibrio se establece ciclos de hidratación (noche) y deshidratación (dia), llegándose a tener velocidades de secado negativas (Cuadros $\mathrm{N}^{\circ} 07$ y 08 ).

\section{GRAFICO N06}
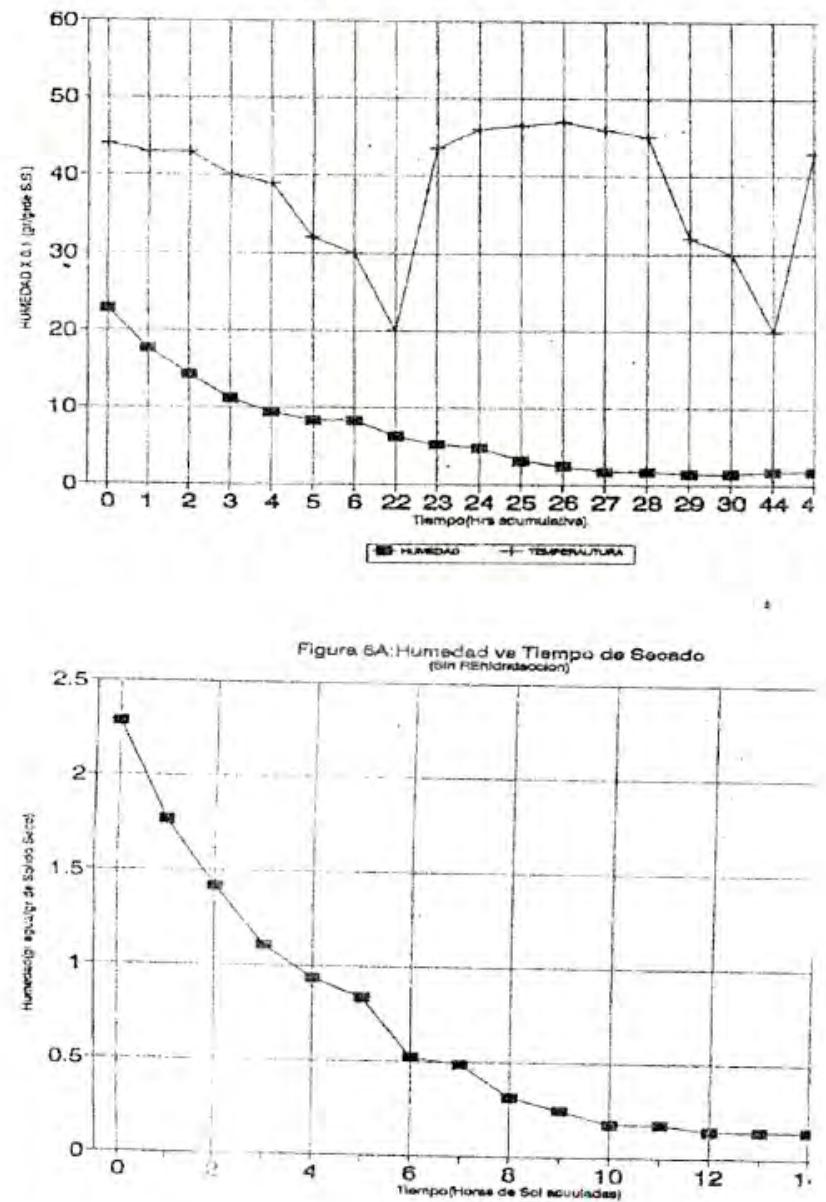

Cuadro № 06: Evaluación del Secado de Lúcuma

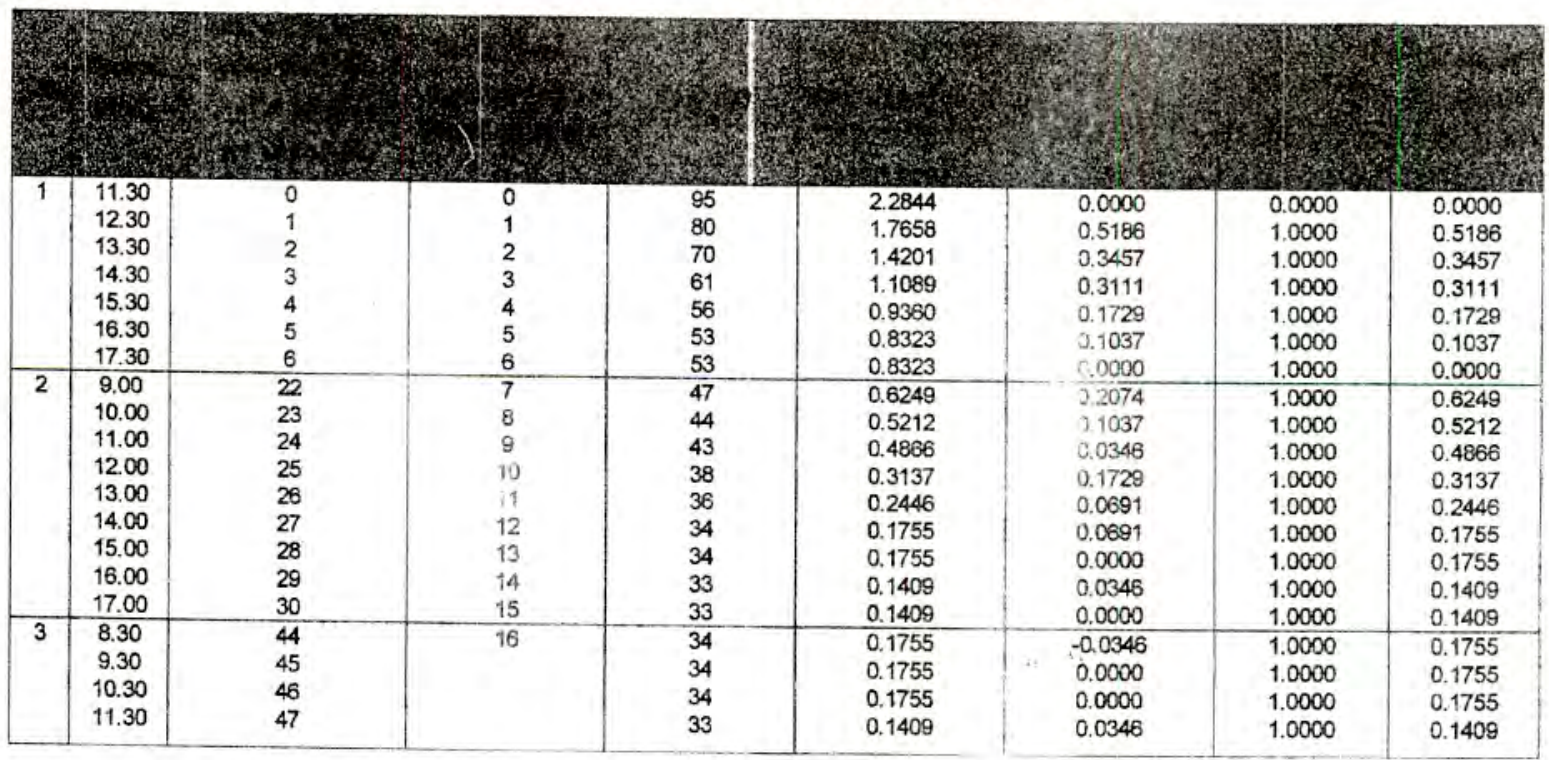



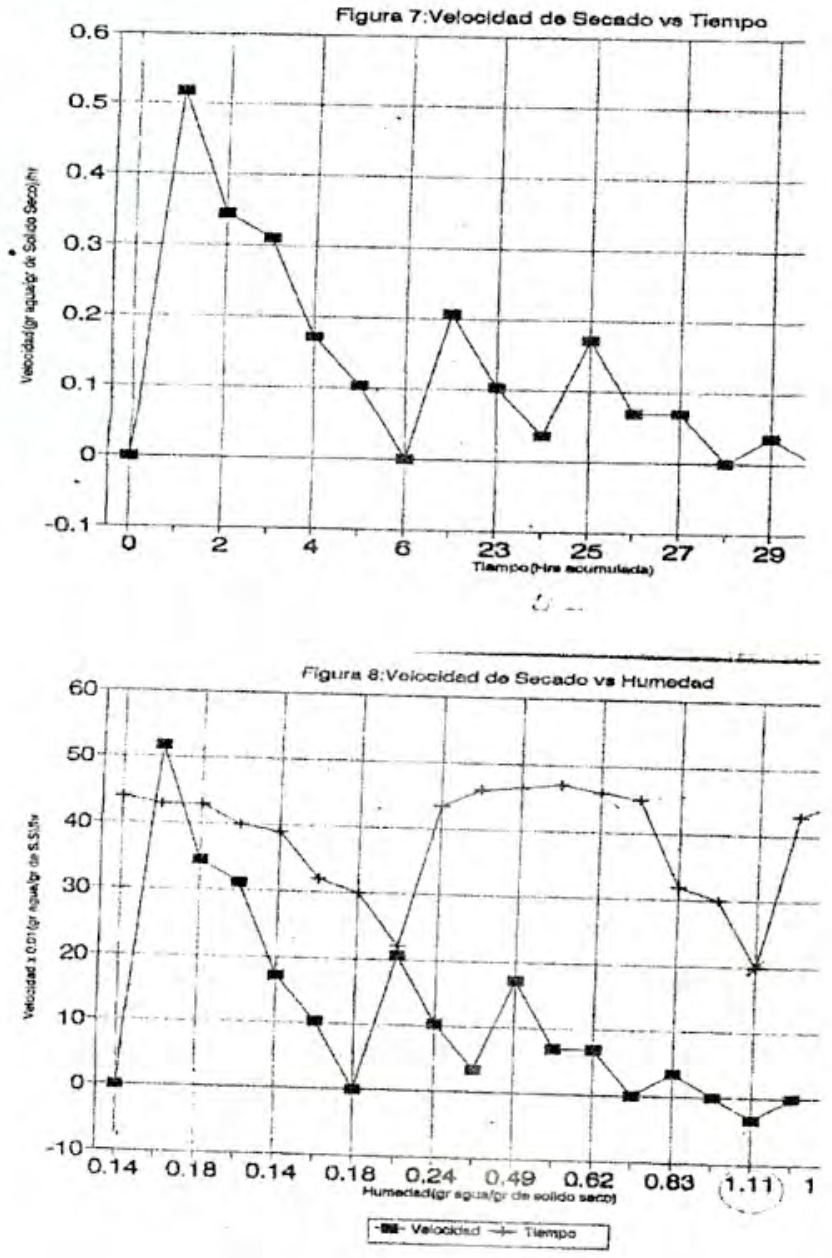

Observando puntos de la curva de velocidad de secado vs tiempo de secado (figura $\mathrm{N}^{a} 8$ ) se nota que es muy dificil determinar en que punto de la curva se dan diferentes tipos de velocidades: Constante, decrecientes debido en partes a características de la dada por la aleotoriedad de los factores del secado y la complejidad de la composición de lúcuma.

Se ha adicionado a la figura $N^{0} 7$, la curva de temperaturas en la cámara de secado, observándose, que para la eliminación del agua de la rehidratación se alcanza velocidades de secado mas altas que para la misma temperatura a la misma hora del dia.

Cuadro No 7: Resultados del Examen Organoléptico Muestra A: Muestra de harina de lúcuma secada sin adición de antioxidante.

Muestra B: Muestra obtenida por secado tradicional.

\begin{tabular}{ccccc}
\hline $\begin{array}{c}\text { Panelista } \\
N^{\circ}\end{array}$ & A & Muestras & B & Total \\
\hline 1 & 4 & 3 & 7 \\
2 & 4 & 3 & 7 \\
3 & 4 & 4 & 8 \\
4 & 1 & 4 & 5 \\
5 & 4 & 3 & 8 \\
6 & 4 & 3 & 7 \\
7 & 4 & 2 & 7 \\
8 & 3 & 3 & 5 \\
9 & 4 & 29 & 7 \\
\hline Total Promedio & 32 & 3.2222 & 61 \\
\hline
\end{tabular}

En cuanto a las características organolépticas del producto final se observó que seguia teniendo el color, olor, sabor característico, pero la textura era dura.

El porcentaje de humedad final alcanzado fue de $9,26 \%$ (base húmeda) lo que comparándolo con los valores que Bergeret (1952) señala para algunas frutas $(20-24 \%)$, se puede indicar como un valor excedente.

Evaluando el rendimiento de la materia prima en harina se tiene los siguiente:

$$
\% R=(33 / 95) \times 1 \mathrm{~kg}=3,47 \mathrm{~kg} \times 100=34.7 \%
$$

Los resultados de la evaluación organoléptica realizada por el panel semi entrenado, y el análisis de varianza se muestra en el cuadro 7 y 8 respectivamente e indican que no existen diferencias significativas entre el sabor de las muestras obtenidas por secado solar tradicional y el secado solar técnico a un nivel de significancia del $0,05 \%$.

\section{Cuadro $N^{\circ}$ 8: Análisis de Varianza}

\begin{tabular}{cccccc}
\hline $\begin{array}{c}\text { Fuente de } \\
\text { Variación }\end{array}$ & $\begin{array}{c}\text { Gracio de } \\
\text { Licartad }\end{array}$ & $\begin{array}{c}\text { Suma de } \\
\text { Cuadrados }\end{array}$ & $\begin{array}{c}\text { Cuadrado } \\
\text { Medio }\end{array}$ & $\mathrm{Fe}$ & $\mathrm{Ft}$ \\
\hline Tratamientos & 1 & 0.5000 & 0.5000 & 0.5714 & 5.32 \\
Panelistas & 8 & 4.7780 & 0.5985 & 0.684 & 3.44 \\
Error & 8 & 6.9998 & & & \\
Total & 17 & 12.2778 & & & \\
\hline
\end{tabular}

\section{CONCLUSIONES}

En las operaciones preliminares para el secado de Lúcume, el sulfitado no es necesario.

En la recha realizada el presente trabajo de Investigación, las temperaturas ambientales iban desde $24,9^{\circ} \mathrm{C}$ (máxima) a $13.1^{\circ} \mathrm{C}$ (mínima) y en la cámara de secado va desde $47^{\circ} \mathrm{C}$ (máxima) y $20^{\circ} \mathrm{C}$ (mínima) durante las horas de sol; pero, la temperatura mínima de trabajo se consideró como $30^{\circ} \mathrm{C}$.

La velocidad del aire de secado a la salida del secador fue casi nula.

La duración del proceso de secado de Lúcuma en esta época (primavera) fue de aproximadamente de 30 hrs o dos dias 015 horas de exposición solar.

El producto obtenido por el método de secado solar técnico conservó las características organolépticas del producto fresco, excepto que la textura de pastosa de fruta pasa a dura.

De los resultados de la evaluación organoléptica se puede concluir que no existe diferencia significativa del sabor entre. la muestra obtenida por Secado Solar Tradicional y Secado Solar Técnico.

El porcentaje de humedad final obtenido para el secado solar técnico fue adecuado.

Las diferentes velocidades de secado son dificiles de determinar por las características de la complejidad de los alimentos y aleatoriedad de los factores de 
secado.

En valores de humedad cercanos a la Humedad de Equilibrio del producto se establece una serie de estados de hidratación (noche) y deshidratación (dia).

\section{REFERENCIA BIBLIOGRÁFICA}

1. Salas W. (1990), copias del curso de envases y empaque de alimentos, FIAL UNALM.

2. Caraso Inés (1990), La necesidad del Secado de Productos agricolas y la alimentación en la Política Nacional del Perú, II Seminario de secado solar del Perú, Cuzco. PSS

3. Aliaga O., Jorge . Néctar, jalea, trozos de lúcuma en almíbar T.A.P:A. U.N.A.L.M.- Perú.

4. Álvarez y Legues (1983), Informe Técnico del Protecto: Desarrollo de Modelo de Deshidratación de Agroproductos, $\mathrm{N}^{\circ} 75-83$, Fondecyt, Chile.

5. A.O.A.C. (1980). Official Methods of analysis. USA

6. Brennan et al (1980), Las operaciones en la ingeniería de Alimentos, Ed. Acribia S.A. Zaragoza España.

7. Brenner K., Manuel (1965), Ensayo de deshidratación de lúcuma (Lúcuma obovata H.P.K.), Tesis UNALM

8. Broker Donald, et al. Drying Cereal Grains (1972). The AVI Publishing Company inc. USA

9. Bsoman Daniela. Deshidratación Solar de Melocotón. Tesis UNALM.

10. Calzada Benza (1980), 143 Frutales nativos Distribuidora el Estudiante. Lima Perú

11. Cobo Bernabé (1891), Historia del Nuevo Mundo, Tomo II, Sevilla España.

12. Cisneros V. Fausto (1959), Contribución al Estudio de la Biología y propagación de la lúcuma (lúcuma obovata) H.P.K., Escueia de Agricultura, La Molina.
13. Fortes, M. \& Olmos, M.R. (1980), Drying Theories: their basisis and limitations as applied to food and grains. In: advices in Drying A.A. Mujumar, Hemisphere Publishing Corporations: 1980 .

14. Geankopolis C. (1986). Procesos de transporte y Operaciones unitarias. Editorial Continental S.A. México.

15. INIDA. (¿??), Informe del Proyecto Lúcuma

16. Proyecto de secado solar (PSS), (i)(\&), informe técnico N²1.G.-UNI, Perú

17. Proyecto de secado solar (PSS) (1986) informe técnico NN4 Teoría Básica del secado solar I.G.I.-UNI-Lima, Perú.

18. Rebhole Thomas (1988), datos sobre Procesos físicos y químicos durante el proceso de secado de alimentos taller de secado de productos agrícolas, PSS, La Molina.

19. Rossi Silvio \& Roa Gonzalo (1980), Instituto de Física Unicamp, Brasil

20. SENAIM (1992), Planillas de Información tecnológica, Dirección Regicical SENAIM - Tacna-Moquegua. Estación Calana, Estación "Los Fichones".

21. Saenz Caimen, et al (1988), Obtención de Manzanas deshidratadas tipo "Crocante" en. Alimentos vol. $12 \mathrm{~N}^{\circ} 2$ Chile.

22. Babilon V. The Origin, variation, immunity and bending of cultivated piant. Charm botanicals. Vol 13. Wathman. 